\title{
NONPARALLEL HYPERPLANES SUPPORT VECTOR REGRESSOR
}

\author{
Ming Hou and Liya Fan
}

School of Mathematics Sciences, Liaocheng University, 252059, P. R. China

\begin{abstract}
Motivated by nonparallel hyperplanes support vector machine (NHSVM), a new regression method of data, named as nonparallel hyperplanes support vector regression (NHSVR), is proposed in this paper. The advantages of NHSVR have two aspects, one is considering the minimization of structure risk by introducing a regularization term in objective function, and another is finding two nonparallel hyperplanes by solving a combined quadratic programming problem. In order to verify the effectiveness of the propose method, a series of comparative experiments are performed with TSVR, LTSVR, and $\varepsilon$-TSVR on five evaluation indexes. The experiment results show that the proposed NHSVR is an effective and efficient algorithm for regression of data.
\end{abstract}

Keywords: twin support vector regression, evaluation index, nonparallel hyperplanes, structural risk.

${ }^{*}$ Corresponding author.

E-mail address: fanliya63@126.com (Liya Fan).

Copyright (C) 2015 Scientific Advances Publishers

2010 Mathematics Subject Classification: 68W40, 68Q25.

Submitted by Jianqiang Gao.

Received February 2, 2015 


\section{Introduction}

Support vector machine (SVM) [1-4], as an excellent kernel-based tool for data classification and regression, has already successfully applied in a wide variety of fields [5-8]. For the classical SVM, the optimal hyperplane can be obtained by maximizing the margin between two parallel boundary hyperplanes, which involves the minimization of a quadratic programming problem (QPP).

Different from SVM, the classification methods of data by two nonparallel hyperplanes were introduced by Bradley and Mangasarian [9] and Mangasarian and Wild [10] with $k$-plane clustering method and generalized eigenvalue proximal support vector machine classifier (GEPSVM), respectively. In both the approaches, a pattern is assigned to a class by the proximity of it to one of the nonparallel hyperplanes. Motivated by GEPSVM, recently, Jayadeva and Chandra [11] presented a twin support vector machine (TSVM) and Shao et al. [12] proposed a nonparallel hyperplanes support vector machine (NHSVM) for binary classification, in which two nonparallel hyperplanes are generated such that each plane is closer to one of two classes and is as far as possible from the other. A main difference between TSVM and NHSVM is that in TSVM the two nonparallel hyperplanes are constructed separately by solving two QPPs and in NHSVM they are constructed simultaneously by solving a single QPP.

Working on similar lines as TSVM, Peng [13] introduced a nonparallel hyperplanes regressor, termed as twin support vector regression (TSVR), which aims at generating two nonparallel functions so that each function determines the $\varepsilon$-insensitive down- or up-bound of the unknown regressor. Similar to TSVM, TSVR also solves two smaller QPPs instead of solving a large one as in the classical SVR [14]. In SVR, the QPP has two groups of constraints for all data points, but in TSVR, only one group of constraints for all data points are used in each QPP. This strategy of solving two smaller QPPs, rather than one large QPP, 
makes TSVR work faster than standard SVR. Recently, Shao et al. [15] extended TSVR to $\varepsilon$-twin support vector regression ( $\varepsilon$-TSVR) by adding extra regularization terms in objective function, which results in the minimization of structural risk. Balasundaram and Tanveer [16] generalized TSVR to Lagrangian twin support vector regression (LTSVR), in which the square of the 2-norm of the vectors of slack variables in TSVR are replaced by 1-norm.

Motivated by the above works, in this paper, we will propose a new nonparallel hyperplanes regressor, termed as nonparallel hyperplanes support vector regression (NHSVR). Although similar to NHSVM, NHSVR also derives two nonparallel hyperplanes, it is completely different from NHSVM. Firstly, NHSVM is used to construct a classifier for the classification of data and NHSVR is used to construct a regressor for fitting, approximation, and prediction of data. Secondly, in NHSVM, all patterns are divided into two classes and appear in the constraints at the same time, while in NHSVR, all patterns are seen as one class and appear in the constraints. Thirdly, NHSVM finds two nonparallel hyperplanes so that each hyperplane is closer to one of the two classes and is as far as possible from the other, whereas NHSVR finds the $\varepsilon$-insensitive up- and down-bound functions for the end regressor. In addition, NHSVR is also different from nonparallel SVR methods, such as TSVR, E-TSVR, LTSVR and so on. This is because in nonparallel SVR methods, two nonparallel hyperplanes are constructed separately by solving two QPPs, but in NHSVR, they are constructed simultaneously by solving a single QPP. Furthermore, in NHSVR, an extra regularization term is added in order to avoid the singular matrix.

In order to verify the effectiveness of NHSVR proposed in the paper, we will perform a series of comparative experiments with TSVR, $\varepsilon$-TSVR, and LTSVR by means of Auto-Mpg (AM), Auto price (AP), Boston housing (BH), Machine CUP (MC), Wisconsin BC (WBC) five datasets taken from UCI database [17]. 
The paper is organized as follows: In Section 2, we briefly recall TSVR and NHSVM. In Section 3, we introduce NHSVR with both linear and nonlinear cases. In Section 4, we perform a series of comparative experiments and in Section 5, we give some concluding remarks.

\section{Background}

\subsection{Twin support vector regression}

In this subsection, we briefly recall TSVR, for details, see [12]. For a given sample set of data $T=\left\{\left(x_{i}, y_{i}\right)\right\}_{i=1}^{n}$, where $x_{i} \in R^{d}$ and $y_{i} \in R$ are input and output of $i$-th sample, respectively, we denote by $A=\left[x_{1}, \cdots, x_{n}\right]^{T} \in R^{n \times d}$ the input matrix and by $Y=\left(y_{1}, \cdots, y_{n}\right)^{T} \in R^{n}$ the output vector. Let $e=(1, \cdots, 1)^{T} \in R^{n}$.

For the linear case, TSVR finds two nonparallel functions $f_{1}(x)=w_{1}^{T} x+b_{1}$ and $f_{2}(x)=w_{2}^{T} x+b_{2}$ by solving a pair of QPPs:

$$
\begin{gathered}
\min _{w_{1}, b_{1}, \xi} \frac{1}{2}\left\|Y-e \varepsilon_{1}-\left(A w_{1}+e b_{1}\right)\right\|^{2}+c_{1} e^{T} \xi \\
\text { s.t. } \quad Y-\left(A w_{1}+e b_{1}\right) \geq e \varepsilon_{1}-\xi, \quad \xi \geq 0, \\
\min _{w_{2}, b_{2}, \eta} \frac{1}{2}\left\|Y+e \varepsilon_{2}-\left(A w_{2}+e b_{2}\right)\right\|^{2}+c_{2} e^{T} \eta \\
\text { s.t. } \quad\left(A w_{2}+e b_{2}\right)-Y \geq e \varepsilon_{2}-\eta, \quad \eta \geq 0,
\end{gathered}
$$

where $c_{1}, c_{2}, \varepsilon_{1}, \varepsilon_{2}>0$ are the parameters given by users, $\xi, \eta \in R^{n}$ are slack vectors and $w_{1}, w_{2} \in R^{d}, b_{1}, b_{2} \in R$ are decision variables, so that each one determines an $\varepsilon$-insensitive down- or up-bound of the end regressor. By solving the following Wolfe dual forms of the problems (1) and (2), respectively, 


$$
\begin{aligned}
& \max _{\alpha} \frac{1}{2} \alpha^{T} G\left(G^{T} G\right)^{-1} G^{T} \alpha+f^{T} G\left(G^{T} G\right)^{-1} G^{T} \alpha-f^{T} \alpha \\
& \text { s.t. } 0 \leq \alpha \leq c_{1} e, \\
& \max _{\beta} \frac{1}{2} \beta^{T} G\left(G^{T} G\right)^{-1} G^{T} \beta+h^{T} G\left(G^{T} G\right)^{-1} G^{T} \beta-h^{T} \beta \\
& \text { s.t. } 0 \leq \beta \leq c_{2} e,
\end{aligned}
$$

where $G=[A, e] \in R^{n \times(d+1)}, f=Y-e \varepsilon_{1} \in R^{n}$, and $h=Y+e \varepsilon_{2} \in R^{n}$, we can get the optimal Lagrangian multipliers $\alpha^{*}$ and $\beta^{*}$ and then deduce that

$$
\begin{aligned}
& {\left[\left(w_{1}^{*}\right)^{T} b_{1}^{*}\right]^{T}=\left(G^{T} G\right)^{-1} G^{T}\left(f-\alpha^{*}\right),} \\
& {\left[\left(w_{2}^{*}\right)^{T} b_{2}^{*}\right]^{T}=\left(G^{T} G\right)^{-1} G^{T}\left(h+\beta^{*}\right) .}
\end{aligned}
$$

It notes that since $G^{T} G$ is a symmetric nonnegative definite matrix, we can regularized it if $G^{T} G$ is singular, that is, we can replace $G^{T} G$ by $G^{T} G+\delta I_{d+1}$, where $\delta>0$ is a sufficiently small number and $I_{d+1}$ is the $d+1$ order unit matrix. Consequently, the estimated regressor can be constructed by

$$
f(x)=\frac{1}{2}\left(f_{1}(x)+f_{2}(x)\right)=\frac{1}{2}\left(w_{1}^{*}+w_{2}^{*}\right)^{T} x+\frac{1}{2}\left(b_{1}^{*}+b_{2}^{*}\right) .
$$

For the nonlinear case, by introducing a kernel function $k: R^{d} \times R^{d} \rightarrow R$ with the reproducing kernel Hilbert space (RKHS) $H$ and the nonlinear feature mapping $\phi: R^{d} \rightarrow H$, TSVR finds nonparallel functions

$$
\begin{aligned}
& f_{1}(x)=\left[k\left(x_{1}, x\right), \cdots, k\left(x_{n}, x\right)\right] w_{1}+b_{1}, \\
& f_{2}(x)=\left[k\left(x_{1}, x\right), \cdots, k\left(x_{n}, x\right)\right] w_{2}+b_{2},
\end{aligned}
$$


by solving the following a pair of QPPs:

$$
\begin{gathered}
\min _{w_{1}, b_{1}, \xi} \frac{1}{2}\left\|Y-e \varepsilon_{1}-\left(K w_{1}+e b_{1}\right)\right\|^{2}+c_{1} e^{T} \xi \\
\text { s.t. } \quad Y-\left(K w_{1}+e b_{1}\right) \geq e \varepsilon_{1}-\xi, \quad \xi \geq 0, \\
\min _{w_{2}, b_{2}, \eta} \frac{1}{2}\left\|Y+e \varepsilon_{2}-\left(K w_{2}+e b_{2}\right)\right\|^{2}+c_{2} e^{T} \eta \\
\text { s.t. } \quad\left(K w_{2}+e b_{2}\right)-Y \geq e \varepsilon_{2}-\eta, \quad \eta \geq 0,
\end{gathered}
$$

where $w_{1}, w_{2} \in R^{n}$ and $K=\left[k\left(x_{i}, x_{j}\right)\right] \in R^{n \times n}$ is the kernel matrix.

Similar to the linear case, by solving the Wolfe dual forms of the problems (5) and (6), respectively, we can deduce that

$$
\begin{aligned}
& {\left[\left(w_{1}^{*}\right)^{T} b_{1}^{*}\right]^{T}=\left(H^{T} H\right)^{-1} H^{T}\left(f-\alpha^{*}\right),} \\
& {\left[\left(w_{2}^{*}\right)^{T} b_{2}^{*}\right]^{T}=\left(H^{T} H\right)^{-1} H^{T}\left(h+\beta^{*}\right),}
\end{aligned}
$$

where $H=[K, e] \in R^{n \times(n+1)}$.

\subsection{Nonparallel hyperplane support vector machine}

In this subsection, we briefly recall NHSVM, for details, see [13]. Let $T=\left\{\left(x_{i}, y_{i}\right)\right\}_{i=1}^{n}$ be a given sample set of data, where $x_{i} \in R^{d}$ and $y_{i} \in\{-1,1\}$ are input and class label of $i$-th sample, respectively. Let

$X_{+}=\left[x_{1}^{+}, \cdots, x_{n_{1}}^{+}\right]^{T} \in R^{n_{1} \times d}$ and $X_{-}=\left[x_{1}^{-}, \cdots, x_{n_{2}}^{-}\right]^{T} \in R^{n_{2} \times d}$ be the input matrices of positive class and negative class, respectively, and $Y=\left(y_{1}, \cdots, y_{n}\right)^{T} \in R^{n}$ be the label vector, where $n_{1}$ and $n_{2}$ are the numbers of the positive samples and negative samples, respectively, and $n_{1}+n_{2}=n$. Let $e_{+} \in R^{n_{1}}, e_{-} \in R^{n_{2}}$, and $e \in R^{n}$ be vectors with elements being ones. 
For the linear case, NHSVM seeks two nonparallel hyperplanes $f_{+}(x)=w_{+}^{T} x+b_{+}=0$ and $f_{-}(x)=w_{-}^{T} x+b_{-}=0$ by considering the following QPP:

$$
\begin{array}{r}
\min _{w_{ \pm}, b_{ \pm}, \xi_{ \pm}} \frac{1}{2}\left(\left\|w_{+}\right\|^{2}+b_{+}^{2}+\left\|w_{-}\right\|^{2}+b_{-}^{2}\right)+\frac{c_{1}}{2}\left(\left\|X_{+} w_{+}+e_{+} b_{+}\right\|^{2}\right. \\
\left.+\left\|X_{-} w_{-}+e_{-} b_{-}\right\|^{2}\right)+c_{2}\left(e_{+}^{T} \xi_{+}+e_{-}^{T} \xi_{-}\right) \\
\text {s.t. } \quad X_{+} w_{+}+e_{+} b_{+}-X_{-} w_{-}-e_{-} b_{-} \geq e_{+}-\xi_{+}, \quad \xi_{+} \geq 0, \\
\quad X_{-} w_{-}+e_{-} b_{-}-X_{+} w_{+}-e_{+} b_{+} \geq e_{-}-\xi_{-}, \quad \xi_{-} \geq 0,
\end{array}
$$

where $c_{1}, c_{2}>0$ are the parameters given by users, $\xi_{+} \in R^{n_{1}}, \xi_{-} \in$ $R^{n_{2}}$ are slack vectors and $w_{+}, w_{-} \in R^{d}, b_{+}, b_{-} \in R$ are decision variables, so that hyperplanes $f_{+}(x)=0$ and $f_{-}(x)=0$ are closer to the positive and negative class and far away from the other class to some extent, respectively. A new input is closer to which, each hyperplane will be assigned to its corresponding class. By solving the Wolfe dual form of the problem (7)

$$
\begin{aligned}
& \max _{\alpha} \frac{1}{2} \alpha^{T} \bar{X}^{T}\left[\left(I+c_{1} \bar{X}_{+}^{T} \bar{X}_{+}\right)^{-1}+\left(I+c_{1} \bar{X}_{-}^{T} \bar{X}_{-}\right)^{-1}\right] \bar{X} \alpha+e^{T} \alpha \\
& \text { s.t. } \quad 0 \leq \alpha \leq c_{2} e
\end{aligned}
$$

where $\bar{X}_{+}=\left[X_{+}, e_{+}\right] \in R^{n_{1} \times(d+1)}, \bar{X}_{-}=\left[X_{-}, e_{-}\right] \in R^{n_{2} \times(d+1)}, \bar{X}=\left[\bar{X}_{+}^{T}\right.$, $\left.\bar{X}_{-}^{T}\right] \in R^{d \times n}$, and $\alpha=\left[\left(\alpha_{+}\right)^{T},\left(\alpha_{-}\right)^{T}\right]^{T} \in R^{n}$, we can obtain the optimal Lagrangian multipliers $\alpha^{*}=\left[\left(\alpha_{+}^{*}\right)^{T},\left(\alpha_{-}^{*}\right)^{T}\right]^{T}$ and then deduce that

$$
\begin{aligned}
& {\left[\left(w_{+}^{*}\right)^{T} b_{+}^{*}\right]^{T}=\left(I+c_{1} \bar{X}_{+}^{T} \bar{X}_{+}\right)^{-1}\left(\bar{X}_{+}^{T} \alpha_{+}^{*}-\bar{X}_{-}^{T} \alpha_{-}^{*}\right),} \\
& {\left[\left(w_{-}^{*}\right)^{T} b_{-}^{*}\right]^{T}=\left(I+c_{1} \bar{X}_{-}^{T} \bar{X}_{-}\right)^{-1}\left(\bar{X}_{-}^{T} \alpha_{-}^{*}-\bar{X}_{+}^{T} \alpha_{+}^{*}\right) .}
\end{aligned}
$$


A new input $x \in R^{d}$ is assigned to the positive or negative class, depending on which of the two hyperplanes it is closer to, i.e.,

$$
\text { class } i=\arg \min _{k=+,-} \frac{\left|\left(w_{k}^{*}\right)^{T} x+b_{k}^{*}\right|}{\left\|w_{k}^{*}\right\|},
$$

where $\mid \cdot$ denotes the absolute value.

For the nonlinear case, by introducing a kernel function $k: R^{d} \times R^{d} \rightarrow R$ with the reproducing kernel Hilbert space (RKHS) $H$ and the nonlinear feature mapping $\phi: R^{d} \rightarrow H$, NHSVM seeks two nonparallel hyperplanes

$$
\begin{aligned}
& f_{+}(x)=\left[k\left(x_{1}, x\right), \cdots, k\left(x_{n}, x\right)\right] w_{+}+b_{+}=0, \\
& f_{-}(x)=\left[k\left(x_{1}, x\right), \cdots, k\left(x_{n}, x\right)\right] w_{-}+b_{-}=0,
\end{aligned}
$$

by considering the following QPP:

$$
\begin{gathered}
\min _{w_{ \pm}, b_{ \pm}, \xi_{ \pm}} \frac{1}{2}\left(\left\|w_{+}\right\|^{2}+b_{+}^{2}+\left\|w_{-}\right\|^{2}+b_{-}^{2}\right)+\frac{c_{1}}{2}\left(\left\|K\left(X_{+}, X\right) w_{+}+e_{+} b_{+}\right\|^{2}\right. \\
\left.+\left\|K\left(X_{-}, X\right) w_{-}+e_{-} b_{-}\right\|^{2}\right)+c_{2}\left(e_{+}^{T} \xi_{+}+e_{-}^{T} \xi_{-}\right) \\
\text {s.t. } \quad K\left(X_{+}, X\right) w_{+}+e_{+} b_{+}-K\left(X_{-}, X\right) w_{-}-e_{-} b_{-} \geq e_{+}-\xi_{+}, \quad \xi_{+} \geq 0, \\
K\left(X_{-}, X\right) w_{-}+e_{-} b_{-}-K\left(X_{+}, X\right) w_{+}-e_{+} b_{+} \geq e_{-}-\xi_{-}, \quad \xi_{-} \geq 0,
\end{gathered}
$$

where $c_{1}, c_{2}>0$ are the parameters given by users and

$$
K\left(X_{+}, X\right)=\left[\begin{array}{ccc}
k\left(x_{1}^{+}, x_{1}\right) & \ldots & k\left(x_{1}^{+}, x_{n}\right) \\
\vdots & \ddots & \vdots \\
k\left(x_{n_{1}}^{+}, x_{1}\right) & \ldots & k\left(x_{n_{1}}^{+}, x_{n}\right)
\end{array}\right] \in R^{n_{1} \times n}
$$




$$
K\left(X_{-}, X\right)=\left[\begin{array}{ccc}
k\left(x_{1}^{-}, x_{1}\right) & \ldots & k\left(x_{1}^{-}, x_{n}\right) \\
\vdots & \ddots & \vdots \\
k\left(x_{n_{2}}^{-}, x_{1}\right) & \ldots & k\left(x_{n_{2}}^{-}, x_{n}\right)
\end{array}\right] \in R^{n_{2} \times n} .
$$

With the similar way in Subsection 2.2, we can get that

$$
\begin{aligned}
& {\left[\left(w_{+}^{*}\right)^{T} b_{+}^{*}\right]^{T}=\left(I+c_{1} \bar{X}_{+}^{T} \bar{X}_{+}\right)^{-1}\left(\bar{X}_{+}^{T} \alpha_{+}^{*}-\bar{X}_{-}^{T} \alpha_{-}^{*}\right),} \\
& {\left[\left(w_{-}^{*}\right)^{T} b_{-}^{*}\right]^{T}=\left(I+c_{1} \bar{X}_{-}^{T} \bar{X}_{-}\right)^{-1}\left(\bar{X}_{-}^{T} \alpha_{-}^{*}-\bar{X}_{+}^{T} \alpha_{+}^{*}\right),}
\end{aligned}
$$

where $\bar{X}_{+}=\left[K\left(X_{+}, X\right), e_{+}\right] \in R^{n_{1} \times(n+1)}, \bar{X}_{-}=\left[K\left(X_{-}, X\right), e_{-}\right] \in R^{n_{2} \times(n+1)}$, $\bar{X}=\left[\bar{X}_{+}^{T}, \bar{X}_{-}^{T}\right]^{T} \in R^{n \times(n+1)}$, and $\alpha=\left[\alpha_{+}^{T}, \alpha_{-}^{T}\right]^{T} \in R^{n}$. A new input $x \in R^{d}$ is assigned to the positive or negative class depending on which of the two hyperplanes it is closer to, i.e.,

$$
\operatorname{class} i=\arg \min _{k=+,-} \frac{\left|K(x, X) w_{k}^{*}+b_{k}^{*}\right|}{\sqrt{w_{k}^{T} K\left(X, X^{T}\right) w_{k}}},
$$

where $K(x, X)=\left[k\left(x_{1}, x\right), \cdots, k\left(x_{n}, x\right)\right]$ and $K=\left[k\left(x_{i}, x_{j}\right)\right] \in R^{n \times n}$ is the kernel matrix.

\section{Nonparallel Hyperplane Support Vector Regression (NHSVR)}

In this section, we study the regression problems based on NHSVM and propose a new regression method, termed as nonparallel hyperplane support vector regression (NHSVR) with linear and nonlinear versions. All symbols used in this section are same as in Subsection 2.1 unless the special instructions.

\subsection{Linear NHSVR}

The basic idea of linear NHSVR is to seek two nonparallel functions $f_{1}(x)=w_{1}^{T} x+b_{1}$ and $f_{2}(x)=w_{2}^{T} x+b_{2}$ such that the functions $f_{1}(x)$ and $f_{2}(x)$ determine the $\varepsilon_{1}$-insensitive down-bound and the $\varepsilon_{2}$-insensitive 
up-bound of the unknown regressor, respectively, and at the same time, the distance between up- and down-bound is the smaller the better. Moreover, we wish the absolute values $\left|y_{i}-\varepsilon_{1}-f_{1}\left(x_{i}\right)\right|,\left|y_{i}+\varepsilon_{2}-f_{2}\left(x_{i}\right)\right|$, and $\left|\left(y_{i}-\varepsilon_{1}-f_{1}\left(x_{i}\right)\right)-\left(y_{i}+\varepsilon_{2}-f_{2}\left(x_{i}\right)\right)\right|, i=1, \cdots, n$ being the smaller the better, and consider the following QPP:

$$
\begin{array}{cl}
\min _{w_{1}, b_{1}, w_{2}, b_{2}, \xi, \eta} \frac{1}{2}\left(\left\|Y-e \varepsilon_{1}-\left(A w_{1}+e b_{1}\right)\right\|^{2}+\left\|Y+e \varepsilon_{2}-\left(A w_{2}+e b_{2}\right)\right\|^{2}\right) \\
+\frac{c_{1}}{2}\left(\left\|w_{1}\right\|^{2}+b_{1}^{2}+\left\|w_{2}\right\|^{2}+b_{2}^{2}\right)+c_{2}\left(e^{T} \xi+e^{T} \eta\right) \\
\text { s.t. } \quad\left[Y-e \varepsilon_{1}-\left(A w_{1}+e b_{1}\right)\right]-\left[Y+e \varepsilon_{2}-\left(A w_{2}+e b_{2}\right)\right] \geq-\eta, \\
& {\left[Y+e \varepsilon_{2}-\left(A w_{2}+e b_{2}\right)\right]-\left[Y-e \varepsilon_{1}-\left(A w_{1}+e b_{1}\right)\right] \geq-\xi,} \\
& \xi \geq 0, \eta \geq 0,
\end{array}
$$

where $c_{1}, c_{2}, \varepsilon_{1}, \varepsilon_{2}>0$ are the parameters given by users, $\xi, \eta \in R^{n}$ are slack vectors and $w_{1}, w_{2} \in R^{d}, b_{1}, b_{2} \in R$ are decision variables. In order to ensure the structural risk minimization, we add a regularization term $\frac{c_{1}}{2}\left(\left\|w_{1}\right\|^{2}+b_{1}^{2}+\left\|w_{2}\right\|^{2}+b_{2}^{2}\right)$ in the objective function of the problem (9).

Considering the Lagrangian function of the problem (9)

$$
\begin{aligned}
L\left(w_{1}, w_{2}, b_{1}, b_{2}, \xi, \eta, \alpha_{1}, \alpha_{2}, \beta_{1}, \beta_{2}\right) \\
=\frac{c_{1}}{2}\left(\left\|w_{1}\right\|^{2}+b_{1}^{2}+\left\|w_{2}\right\|^{2}+b_{2}^{2}\right)+\frac{1}{2}\left(\left\|Y-e \varepsilon_{1}-\left(A w_{1}+e b_{1}\right)\right\|^{2}\right. \\
\left.\quad+\left\|Y+e \varepsilon_{2}-\left(A w_{2}+e b_{2}\right)\right\|^{2}\right) \\
\quad+c_{2}\left(e^{T} \xi+e^{T} \eta\right)-\alpha_{1}^{T}\left(\left(Y-e \varepsilon_{1}-\left(A w_{1}+e b_{1}\right)\right)-\left(Y+e \varepsilon_{2}\right.\right. \\
\left.\left.\quad-\left(A w_{2}+e b_{2}\right)\right)+\eta\right)-\alpha_{2}^{T} \eta-\beta_{1}^{T}\left(\left(Y+e \varepsilon_{2}-\left(A w_{2}+e b_{2}\right)\right)\right. \\
\left.\quad-\left(Y-e \varepsilon_{1}-\left(A w_{1}+e b_{1}\right)\right)+\xi\right)-\beta_{2}^{T} \xi,
\end{aligned}
$$


where $\alpha_{1}, \alpha_{2}, \beta_{1}, \beta_{2} \in R_{+}^{n}$ are the vectors of Lagrange multipliers, and letting $\frac{\partial L}{\partial w_{1}}=\frac{\partial L}{\partial w_{2}}=\frac{\partial L}{\partial b_{1}}=\frac{\partial L}{\partial b_{2}}=\frac{\partial L}{\partial \xi}=\frac{\partial L}{\partial \eta}=0$, we can deduce that

$$
\left\{\begin{array}{l}
c_{1} w_{1}-A^{T}\left(Y-e \varepsilon_{1}-\left(A w_{1}+e b_{1}\right)\right)+A^{T} \alpha_{1}-A^{T} \beta_{1}=0, \\
c_{1} b_{1}-e^{T}\left(Y-e \varepsilon_{1}-\left(A w_{1}+e b_{1}\right)\right)+e^{T} \alpha_{1}-e^{T} \beta_{1}=0, \\
c_{1} w_{2}-A^{T}\left(Y+e \varepsilon_{2}-\left(A w_{2}+e b_{2}\right)\right)-A^{T} \alpha_{1}+A^{T} \beta_{1}=0 \\
c_{1} b_{2}-e^{T}\left(Y+e \varepsilon_{2}-\left(A w_{2}+e b_{2}\right)\right)-e^{T} \alpha_{1}+e^{T} \beta_{1}=0, \\
c_{2} e-\beta_{1}-\beta_{2}=0 \Rightarrow 0 \leq \beta_{1} \leq c_{2} e, \\
c_{2} e-\alpha_{1}-\alpha_{2}=0 \Rightarrow 0 \leq \alpha_{1} \leq c_{2} e .
\end{array}\right.
$$

Proceeding to the next step, we have

$$
\begin{gathered}
c_{1}\left[\begin{array}{c}
w_{1} \\
b_{1}
\end{array}\right]-\left[\begin{array}{c}
A^{T} \\
e^{T}
\end{array}\right]\left(Y-e \varepsilon_{1}-[A \quad e]\left[\begin{array}{c}
w_{1} \\
b_{1}
\end{array}\right]\right)+\left[\begin{array}{c}
A^{T} \\
e^{T}
\end{array}\right]\left(\alpha_{1}-\beta_{1}\right)=0, \\
c_{1}\left[\begin{array}{c}
w_{2} \\
b_{2}
\end{array}\right]-\left[\begin{array}{c}
A^{T} \\
e^{T}
\end{array}\right]\left(Y+e \varepsilon_{2}-[A e]\left[\begin{array}{c}
w_{2} \\
b_{2}
\end{array}\right]\right)+\left[\begin{array}{c}
A^{T} \\
e^{T}
\end{array}\right]\left(\beta_{1}-\alpha_{1}\right)=0 .
\end{gathered}
$$

Put $G=[A, e], f=Y-e \varepsilon_{1}, h=Y+e \varepsilon_{2}, u_{1}=\left[w_{1}^{T}, b_{1}\right]^{T}$, and $u_{2}=\left[w_{2}^{T}, b_{2}\right]^{T}$, then we get

$$
\begin{aligned}
& u_{1}=\left(c_{1} I+G^{T} G\right)^{-1} G^{T}\left(f-\alpha_{1}+\beta_{1}\right), \\
& u_{2}=\left(c_{1} I+G^{T} G\right)^{-1} G^{T}\left(h+\alpha_{1}-\beta_{1}\right) .
\end{aligned}
$$

Substituting (10) and (11) into the Lagrangian function, we can obtain the Wolfe dual form of the problem (9)

$$
\begin{aligned}
& \max _{\gamma}-\frac{1}{2} \gamma^{T}\left(2 G\left(c_{1} I+G^{T} G\right)^{-1}\right) \gamma+g^{T} G\left(c_{1} I+G^{T} G\right)^{-1} \gamma-g^{T} \gamma \\
& \text { s.t. } \quad-c_{2} e \leq \gamma \leq c_{2} e,
\end{aligned}
$$


where $\gamma=\alpha_{1}-\beta_{1} \in R^{n}, g=f-h \in R^{n}$ and $I$ is an identity matrix of appropriate dimensions. By solving the problem (12), we can get the optimal solution $\gamma^{*}$ and then

$$
\begin{aligned}
& u_{1}^{*}=\left[\left(w_{1}^{*}\right)^{T}, b_{1}^{*}\right]^{T}=\left(c_{1} I+G^{T} G\right)^{-1} G^{T}\left(f-\gamma^{*}\right), \\
& u_{2}^{*}=\left[\left(w_{2}^{*}\right)^{T}, b_{2}^{*}\right]^{T}=\left(c_{1} I+G^{T} G\right)^{-1} G^{T}\left(h+\gamma^{*}\right) .
\end{aligned}
$$

Therefore, the estimated regressor is $f(x)=\frac{1}{2}\left(f_{1}(x)+f_{2}(x)=\frac{1}{2}\left(w_{1}^{*}+w_{2}^{*}\right)^{T} x\right.$ $+\frac{1}{2}\left(b_{1}^{*}+b_{2}^{*}\right)$.

\subsection{Nonlinear NHSVR}

By introducing a kernel function, we can extend linear NHSVR into nonlinear version. Let $k: R^{d} \times R^{d} \rightarrow R$ be a kernel function with the reproducing kernel Hilbert space (RKHS) $H$ and the nonlinear feature mapping $\phi: R^{d} \rightarrow H$. We denote by $K=\left[k\left(x_{i}, x_{j}\right)\right] \in R^{n \times n}$ the kernel matrix and $K=(x, A)=\left[k\left(x_{1}, x\right), \cdots, k\left(x_{n}, x\right)\right]$. Nonlinear NHSVR seeks two nonparallel hyperplanes $f_{1}(x)=K(x, A) u_{1}+b_{1}$ and $f_{1}(x)=K(x, A) u_{2}+b_{2}$ by considering the following optimization problem:

$$
\begin{aligned}
\min _{u_{1}, b_{1}, u_{2}, b_{2}, \xi, \eta} & \frac{1}{2}\left(\left\|Y-e \varepsilon_{1}-\left(K u_{1}+e b_{1}\right)\right\|^{2}+\left\|Y+e \varepsilon_{2}-\left(K u_{2}+e b_{2}\right)\right\|^{2}\right) \\
+ & \frac{c_{1}}{2}\left(\left\|u_{1}\right\|^{2}+b_{1}^{2}+\left\|u_{2}\right\|^{2}+b_{2}^{2}\right)+c_{2}\left(e^{T} \xi+e^{T} \eta\right) \\
\text { s.t. } \quad & {\left[Y+e \varepsilon_{2}-\left(K u_{2}+e b_{2}\right)\right]-\left[Y-e \varepsilon_{1}-\left(K u_{1}+e b_{1}\right)\right] \geq-\xi, } \\
& {\left[Y-e \varepsilon_{1}-\left(K u_{1}+e b_{1}\right)\right]-\left[Y+e \varepsilon_{2}-\left(K u_{2}+e b_{2}\right)\right] \geq-\eta, } \\
& \xi \geq 0, \eta \geq 0,
\end{aligned}
$$

where $c_{1}, c_{2}, \varepsilon_{1}, \varepsilon_{2} \geq 0$ are the parameters given by users, $\xi, \eta$ are the slack vectors and $u_{1}, u_{2} \in R^{n}, b_{1}, b_{2} \in R$ are decision variables. With 
the similar way in Subsection 3.1, we can obtain the Wolfe dual form of the problem (13)

$$
\begin{aligned}
& \max _{\gamma}-\frac{1}{2} \gamma^{T}\left(2 H\left(c_{1} I+H^{T} H\right)^{-1}\right) \gamma+g^{T} H\left(c_{1} I+H^{T} H\right)^{-1} \gamma-g^{T} \gamma \\
& \text { s.t. } \quad-c_{2} e \leq \gamma \leq c_{2} e,
\end{aligned}
$$

where $H=[K, e]$. By solving the problem (14), we can get the optimal solution $\gamma^{*}$ and then

$$
\begin{aligned}
& v_{1}^{*}=\left[\left(u_{1}^{*}\right)^{T}, b_{1}^{*}\right]^{T}=\left(c_{1} I+H^{T} H\right)^{-1} H^{T}\left(f-\gamma^{*}\right), \\
& v_{2}^{*}=\left[\left(u_{2}^{*}\right)^{T}, b_{2}^{*}\right]^{T}=\left(c_{1} I+H^{T} H\right)^{-1} H^{T}\left(h+\gamma^{*}\right) .
\end{aligned}
$$

Hence, the estimated regressor is $f(x)=\frac{1}{2} K(x, X)\left(u_{1}^{*}+u_{2}^{*}\right)+\frac{1}{2}\left(b_{1}^{*}+b_{2}^{*}\right)$.

\section{Experiments}

In order to demonstrate the effectiveness of the proposed NHSVR, in this section, we perform a series of comparative experiments with TSVR, LTSVR, and $\varepsilon$-TSVR on Auto-Mpg, Auto price, Boston housing, Machine CUP, Wisconsin BC five datasets taken from UCI database. In order to save experimental time and without loss of generality, all experiments are implemented in linear version and in MATLAB (R2010b) on Windows 7 running on a PC.

We know that the performance of these methods seriously depends on the choice of parameters, In order to compare the optimal results, all the regularization parameters of modellings are selected optimally from $\left\{10^{-5}, \cdots, 10^{5}\right\}$ by grid search and take $\varepsilon_{1}=\varepsilon_{2}$. Specifically, for Auto price dataset, take $\varepsilon_{1}=\varepsilon_{2}=10^{-2}, c_{1}=c_{2}=10^{2}, c_{3}=c_{4}=10^{3} \quad$ in TSVR, $\varepsilon$-TSVR and LTSVR and $c_{1}=10^{3}, c_{2}=10^{2}$ in NHSVR; for Auto- 
Mpg dataset, take $\varepsilon_{1}=\varepsilon_{2}=10^{-1}, c_{1}=c_{2}=10^{-3}, c_{3}=c_{4}=1$ in TSVR, $\varepsilon$-TSVR and LTSVR and $c_{1}=1, c_{2}=10^{-3}$ in NHSVR; for Machine CPU dataset, take $\varepsilon_{1}=\varepsilon_{2}=10^{2}, c_{1}=c_{2}=10^{-1}, c_{3}=c_{4}=10 \quad$ in TSVR, $\varepsilon$-TSVR and LTSVR and $c_{1}=1, c_{2}=10^{-1}$ in NHSVR; for Boston housing dataset, take $\varepsilon_{1}=\varepsilon_{2}=10^{-2}, c_{1}=c_{2}=10^{-3}, c_{3}=c_{4}=1$ in TSVR, $\varepsilon$-TSVR and LTSVR and $c_{1}=1, c_{2}=10^{-1}$ in NHSVR; for Wisconsin $\mathrm{BC}$ dataset, take $\varepsilon_{1}=\varepsilon_{2}=1, c_{1}=c_{2}=10^{-2}, c_{3}=c_{4}=1$ in TSVR, $\varepsilon$-TSVR and LTSVR and $c_{1}=1, c_{2}=10^{-2}$ in NHSVR. The fivefold cross-validation method is used in all experiments.

We use MAE, RMSE, SSE, NMSE, and $R^{2}$ five evaluation indexes for evaluating the performance of all algorithms, which are listed in Table 1, where $m$ denotes the number of testing samples, $y_{i}$ and $\hat{y}_{i}$ denote the true value and the predicted value of a testing input $x_{i}$, respectively, and $\bar{y}_{i}=\sum_{i=1}^{m} y_{i}$. It notes that the index $R^{2}$ is the larger the better. 
Table 1. Evaluation indexes

\begin{tabular}{cc}
\hline Name & Expression \\
\hline MAE & RAE $=\frac{1}{m} \sum_{i=1}^{m}\left|y_{i}-\hat{y}_{i}\right|$ \\
\hline RMSE & $S S E=\sqrt{\frac{1}{2} \sum_{i=1}^{m}\left(y_{i}-\hat{y}_{i}\right)^{2}}$ \\
\hline SSE & $S S T=\sum_{i=1}^{m}\left(y_{i}-\hat{y}_{i}\right)^{2}$ \\
\hline SST & $S S R=\sum_{i=1}^{m}\left(\hat{y}_{i}-\bar{y}_{i}\right)^{2}$ \\
\hline NMSE & $N M S E=S S E / S S T=\frac{\sum_{i=1}^{m}\left(y_{i}-\hat{y}_{i}\right)^{2}}{\sum_{i=1}^{m}\left(y_{i}-\bar{y}_{i}\right)^{2}}$ \\
\hline$R^{2}$ & $S S R / S S T=\frac{\sum_{i=1}^{m}\left(\hat{y}_{i}-\bar{y}_{i}\right)^{2}}{\sum_{i=1}^{m}\left(y_{i}-\bar{y}_{i}\right)^{2}}$ \\
\hline & $R^{2}=S$
\end{tabular}

Experiment results are showed in Table 2, where "Time" denotes the running time (second). From Table 2, we can see that for Auto-price dataset, NHSVR is a competitive algorithm compared with TSVR, LTSVR, and $\varepsilon$-TSVR. But the running time of NHSVR is significantly less than that of TSVR, LTSVR, and E-TSVR. For Auto-Mpg and Machine CPU two datasets, NHSVR is obviously better than TSVR, LTSVR, and $\varepsilon$-TSVR both the evaluation index value and the running time. For Boston housing and Wisconsin BC two datasets, NHSVR is better than TSVR, LTSVR, and $\varepsilon$-TSVR on the whole. But the running time of NHSVR is the longest for Wisconsin BC dataset. 
Table 2. Comparison results of four algorithms

\begin{tabular}{|c|c|c|c|c|c|c|c|}
\hline Dataset & Regressor & MAE & RMSE & $\mathrm{SSE}$ & NMSE & $R^{2}$ & Time \\
\hline Auto Price & TSVR & $2.7132 \mathrm{e}+003$ & $3.9081 \mathrm{e}+003$ & $5.9924 \mathrm{e}+008$ & 0.4187 & 1.1206 & 0.9852 \\
\hline \multirow[t]{3}{*}{$(195 * 15)$} & $\varepsilon$-TSVR & $2.8207 \mathrm{e}+003$ & $4.1271 \mathrm{e}+003$ & $6.7064 \mathrm{e}+008$ & 0.4466 & 1.0300 & 0.9288 \\
\hline & LTSVR & $2.7059 e+003$ & $3.9209 \mathrm{e}+003$ & $6.0648 \mathrm{e}+008$ & 0.4275 & 1.0979 & 25.4217 \\
\hline & NHSVR & $2.7804 \mathrm{e}+003$ & $3.9541 \mathrm{e}+003$ & $6.1819 \mathrm{e}+008$ & 0.3961 & 0.9775 & 0.5922 \\
\hline Auto-Mpg & TSVR & 2.9838 & 3.7552 & $1.1746 \mathrm{e}+003$ & 0.4001 & 0.9481 & 1.9882 \\
\hline \multirow[t]{3}{*}{$(398 * 8)$} & $\varepsilon$-TSVR & 2.8451 & 3.6340 & $1.1062 \mathrm{e}+003$ & 0.3760 & 0.9821 & 2.1626 \\
\hline & LTSVR & 3.3221 & 3.8813 & $1.1750 \mathrm{e}+003$ & 0.4491 & 1.6133 & 5.2028 \\
\hline & NHSVR & 2.8294 & 3.6192 & $1.1009 \mathrm{e}+003$ & 0.3740 & 0.9807 & 1.6843 \\
\hline Machine CPU & TSVR & 24.3943 & 40.9018 & $8.2082 \mathrm{e}+004$ & 0.1865 & 1.3836 & 0.8677 \\
\hline \multirow[t]{3}{*}{$(209 * 8)$} & $\varepsilon$-TSVR & 24.2391 & 40.8946 & $8.2228 \mathrm{e}+004$ & 0.1855 & 1.3807 & 0.7331 \\
\hline & LTSVR & 24.4125 & 40.7767 & $8.1416 \mathrm{e}+004$ & 0.1861 & 1.3852 & 1.5464 \\
\hline & NHSVR & 23.8419 & 37.0460 & $6.9712 \mathrm{e}+004$ & 0.1675 & 1.3934 & 0.4730 \\
\hline Boston Housing & TSVR & 4.2426 & 5.8197 & $3.7472 \mathrm{e}+003$ & 0.6401 & 0.9319 & 3.2864 \\
\hline \multirow[t]{3}{*}{$(506 * 14)$} & $\varepsilon$-TSVR & 4.2411 & 5.8182 & $3.7457 \mathrm{e}+003$ & 0.6398 & 0.9317 & 3.2413 \\
\hline & LTSVR & 4.2427 & 5.8197 & $3.7472 \mathrm{e}+003$ & 0.6401 & 0.9319 & 7.7531 \\
\hline & NHSVR & 4.1727 & 5.7299 & $3.6483 \mathrm{e}+003$ & 0.6067 & 0.9029 & 3.9027 \\
\hline Wisconsin BC & TSVR & 0.2677 & 0.3948 & 22.3189 & 0.1817 & 0.8483 & 5.9729 \\
\hline \multirow[t]{3}{*}{$\left(683^{*} 11\right)$} & $\varepsilon$-TSVR & 0.2682 & 0.3943 & 22.2513 & 0.1812 & 0.8544 & 4.7315 \\
\hline & LTSVR & 0.2680 & 0.3948 & 22.3103 & 0.1817 & 0.8477 & 12.7302 \\
\hline & NHSVR & 0.2681 & 0.3943 & 22.2507 & 0.1812 & 0.8544 & 13.7077 \\
\hline
\end{tabular}


By the above analysis, we can conclude that NHSVR proposed in this paper is an effective and efficient algorithm for regression of data.

\section{Conclusion}

Motivated by NHSVM, we propose a new regression method of data in this paper, named as NHSVR with linear and nonlinear versions. The advantages of NHSVR have two aspects, one is considering the minimization of structure risk by introducing a regularization term in objective function, and another is finding two nonparallel hyperplanes by solving a combined quadratic programming problem. In order to verify the effectiveness of NHSVR, we perform a series of comparative experiments with TSVR, LTSVR, and $\varepsilon$-TSVR on five evaluation indexes and five datasets taken from UCI database. We can see from the experiments that although the QPP involved in NHSVR is larger than each of two small QPPs in TSVR, but it consumes less running time than TSVR on Auto-price, Auto-Mpg, and Machine CPU three datasets, and spends almost the same running time on Boston housing dataset. According to the experiment results, we can conclude that NHSVR is an effective and efficient algorithm for regression of data.

\section{References}

[1] V. N. Vapnik, The Natural of Statistical Learning Theory, Springer, New York, 1995.

[2] V. N. Vapnik, Statistical Learning Theory, Wiley, New York, 1998.

[3] C. J. C. Burges, A tutorial on support vector machines for pattern recognition, Data Mining Knowledge Discovery 2(2) (1998), 121-167.

[4] V. Christianini and J. Shawe-Taylor, An Introduction to Support Vector Machines, Cambridge University Press, Cambridge, 2002.

[5] E. Osuna, R. Freund and F. Girosi, Training support vector machines: An application to face detection, in: Proceedings of IEEE Computer Vision and Pattern Recognition, San Juan, Puerto Rico, (1997), 130-136.

[6] T. Joachims, C. Ndellec and C. Rouveriol, Text categorization with support vector machines: Learning with many relevant features, in: European Conference on Machine Learning No. 10, Chemnitz, Germany, (1998), 137-142. 
[7] M. P. S. Brown, W. N. Grundy and D. Lin et al., Knowledge-based analysis of microarray gene expression data by using support vector machine, Proceedings of the National Academy of Sciences of the United States of America 97(1) (2000), 262-267.

[8] H. Ince and T. B. Trafalis, Support Vector Machine for Regression and Applications to Financial Forecasting, in: International Joint Conference on Neural Networks (IJCNN02), IEEE-INNS-ENNS, Como, Italy, 2002.

[9] P. S. Bradley and O. L. Mangasarian, K-plane clustering, J. Glob. Optim. 16(1) (2000), 23-32.

[10] O. L. Mangasarian and E. W. Wild, Multisurface proximal support vector classification via generalize eigenvalues, IEEE Trans. Pattern Anal. Machine Intell. 28(1) (2006), 69-74.

[11] R. Khemchandani Jayadeva and S. Chandra, Twin support vector machines for pattern classification, IEEE Transactions on Pattern Analysis and Machine Intelligence 29(5) (2007), 905-910.

[12] Y. H. Shao, W. J. Chen and N. Y. Deng, Nonparallel hyperplane support vector machine for binary classification problems, Information Sciences 263 (2013), 22-35.

[13] X. Peng, TSVR: An efficient twin support vector machine for regression, Neural Networks 23 (2010), 365-372.

[14] D. Basak, S. Pal and D. C. Patranabis, Support vector regression, Neural Information 10 (2007), 203-224.

[15] Y. H. Shao and C. H. Zhang et al., An $\varepsilon$-twin support vector machine for regressor, Neural Comput. 23 (2013), 175-185.

[16] S. Balasundaram and M. Tanveer, On Lagrangian twin support vector regression, Neural Comput. 22 (2013), 257-267.

[17] http://archive.ics.uci.edu/ml/datasets.html. 